\title{
UHF DTV Band Channel Characterization for Mobile Reception
}

\author{
Assia Semmar ${ }^{\dagger}$, Jean-Yves Chouinard ${ }^{\dagger}$, Xianbin Wang ${ }^{\ddagger}$ and Yiyan $\mathrm{Wu}^{\ddagger}$
}

\begin{abstract}
This paper presents measurement results obtained in the first phase field test campaign carried out in the UHF band in Ottawa with the aim to characterizing mobile reception of digital terrestrial television. The temporal and spatial variations of the impulse response are analyzed. An antenna diversity receiver was used and the signals received from each antenna are then compared and the diversity reception achieved using switched diversity.
\end{abstract}

Index Terms-Digital Terrestrial Television, ATSC-8VSB, Spatial Diversity.

\section{INTRODUCTION}

Since 1995, the Advanced Television System Committee (ATSC) specified the terrestrial digital television standard for the United States [1]. Many countries including Canada adopted the ATSC-8VSB DTV standards. As per the standard, the transmitted signal carries a stream up to 19.39 $\mathrm{Mb} / \mathrm{s}$ over a $6 \mathrm{MHz}$ channel. The standard uses an eightlevel Vestigial Sideband (8VSB) modulation scheme with a pilot signal. Reed-Solomon coding, interleaving and trellis coding are used to provide error protection.

Mobility and portability are two added value characteristics offered by digital terrestrial television (DTV). These two features are not provided by cable or satellite systems. During the past years, various experimental and laboratory tests have shown that mobile reception are feasible using OFDM based system [2], but no studies have been published about mobile reception in the case of the ATSC 8VSB system.

The objective of this study is to characterize the digital television terrestrial propagation channel for mobile applications. An experimental study of the propagation of DTV signals, with a symbol rate of $10.76 \mathrm{Msymbols} / \mathrm{s}$, has been conducted in the Ottawa region. The main problems associated with mobile communications are the Doppler effects and the multipath fading caused by reflection and scattering from obstructions in the vicinity of the receiver.

\footnotetext{
${ }^{\dagger}$ A. Semmar and J.-Y. Chouinard are with the Department of Electrical and Computer Engineering, Laval University, Quebec, Quebec, Canada, G1K 7P4. Email: \{assia,chouinar\}@ gel.ulaval.ca

$¥ \mathrm{X}$. Wang and $\mathrm{Y}$. Wu are with the Communications Research Centre Canada, 3701 Carling Ave., Box. 11490, Station H, Ottawa, Ontario, Canada, K2H 8S2. Email: \{xianbin.wang,yiyan.wu\}@crc.ca
}

An effective way to mitigate the effects of multipath and improve the signal quality is the use of antenna diversity.

This paper is organized as follows. Section II provides a general description of the digital terrestrial television system, ATSC 8-VSB. In section III, the equipment and methodology of subsequent data captures are explained in detail. The results of received signals, estimated channel response, rms delay spread, excess delay and the effect of the spatial diversity are reported in section IV. The conclusions are drawn in section $\mathrm{V}$.

\section{ATSC 8-VSB SYSTEM DESCRIPTION}

The ATSC system was designed to transmit high-quality video and audio (HDTV) and ancillary data over a single $6 \mathrm{MHz}$ channel. The digital terrestrial transmission system is $19.39 \mathrm{Mbs} / \mathrm{s}$ serial data stream comprised of 188-byte MPEG-2 data packets. The incoming data is randomized to ensure energy dispersal and then processed for forward error correction (FEC) in the form of Reed-Solomon coding (RS). Trellis coding and conventional interleaving are applied to the output of the RS encoder for burst noise protection. The trellis code modulator converts the output bits from the RS-encoder to an 8ASK symbol stream with a $2 / 3$ coding rate. These symbols are converted to an analog VSB waveform that can be transmitted via RF amplifiers. A low power pilot is added to signal for carrier recovery [1].

The data randomized and coded packets are formatted into data frames and data fields. Each data frame contains two data fields and each field is divided into 313 segments and shall start with one complete data segment of Data Field Sync as shown in figure 1. The Data Field Sync contains the segment of synchronization, the pseudo-random sequences (PN-511, PN-63) and the VSB mode identification. The PN511 sequence is used as training signal for the receiver's equalizer and will be used in our work to estimate the channel impulse response.

\section{MOBILE FIELD TEST IN OTTAWA}

The measurement campain planning was done according to the objective of characterizing the digital television 


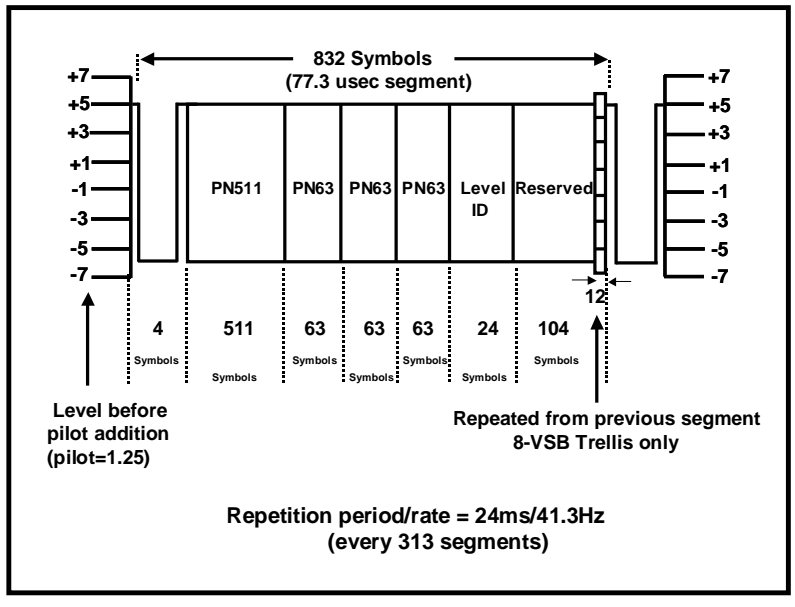

Fig. 1. 8-VSB Data Field Sync. description.

terrestrial propagation channel for mobile applications by means of an analysis of the time and spatial received signal in different environments. The DTV station, covering

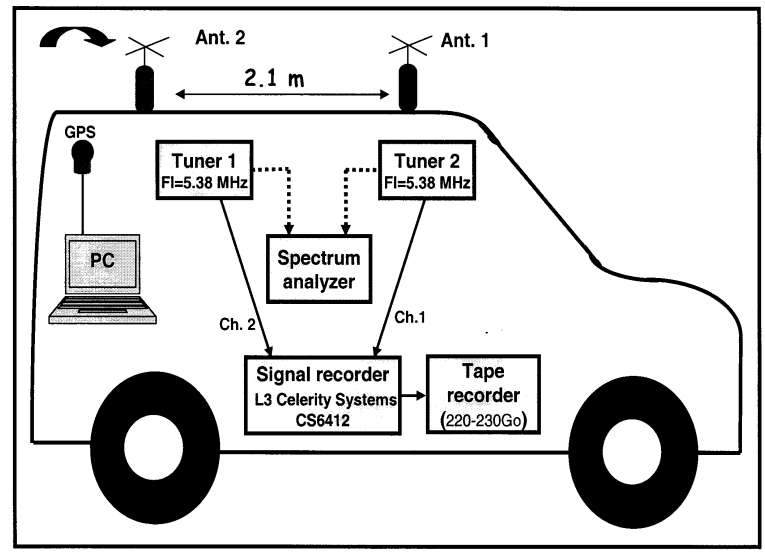

Fig. 2. Bloc diagram of the measurement vehicle.

Ottawa and the surroundings, is located at Manotick about $30 \mathrm{~km}$ south of Ottawa. The measurement signal was transmitted from an omni-directional antenna at a height of $215.4 \mathrm{~m}$ above ground with $30 \mathrm{~kW}$ ERP on UHF channel 67 channel (788-794 MHz) [3]. The channel RF bandwidth is $6 \mathrm{MHz}$. The receiver is installed in a mobile vehicle and connected to two omni-directional antenna on the roof as illustrated in figure 2. The two antennas have identical radiation patterns and polarizations. The 8-VSB signal is received using a professional DTV tuner which brings the signal down to an IF of about 43-47 MHz. The tuner output is downconverted to a lower IF of $5.38 \mathrm{MHz}$, comparable to the VSB symbol rate, filtered and then the envelope of the received signal from each branch is stored on digital tape for computer processing. The mobile measurements have been made in order to record as much received samples as possible along a route with different vehicle speeds. The measurement routes are located about $10 \mathrm{~km}$ from the transmitter and the vehicle speed ranges from 30 to $50 \mathrm{~km} / \mathrm{h}$.

\section{EXPERIMENTALS RESULTS}

\section{A. Channel characterization}

Channel model: Many physical factors in the radio propagation channel including multipath propagation, speed of the mobile and a speed of surroundings objects influence the received signal which may consist of a large number of attenuated, time delayed, phase shifted replicas of the transmitted signal. The channel can be modelled as a linear time variant system [4]. The low pass equivalent channel impulse response can be expressed as:

$$
h(t, \tau)=\sum_{n=0}^{N-1} A_{n}(t) e^{-j \theta_{n}(t)} \delta\left[t-\tau_{n}(t)\right]
$$

where $A_{n}$ and $\tau_{n}$ are the amplitudes and the delays of the $n$th multipath component and $N$ is the number of paths. $\theta_{n}$ is the phase shift. Because of the motion of the vehicule and the surroundings objects, the parameters $A_{n}, \tau_{n}, \theta_{n}$ and $N$ are randomly time-varying functions [5].

Channel estimation: Channel estimation relies entirely on the data-field sync signals, described in 1, and particulary the 511-PN sequence. The data-field sync is transmitted approximately only once every $24.2 \mathrm{~ms}$ and the time duration of the PN sequence is only $47.48 \mu \mathrm{s}$. As result, this portion of recorded data is used for channel estimation. For each recorded data, after downconverting the received signal to the $5.38 \mathrm{MHz}$ IF, we locate the time position of the first 511-PN sequence, and the received signal is passed through an IF noise and interference limiting filter. The channel response is obtained by correlating the baseband filtered signal with a reference signal based on the PN sequence. This approach to estimate the channel response is similar to the sliding correlator technique [4] except that the PN sequence is embedded in the DTV signal.

Figure 3 illustrates a typical mobile channel impulse response in the Chemin LineBank street which represents an open environment. The estimate channel statistics show a variation of the individual impulses responses depending on the number of 8-VSB frames, this can be related to the distance covered by the vehicle. Note that the vehicle speed is about $40 \mathrm{~km} / \mathrm{h}$ and the maximum Doppler frequency is about $f_{m}=v / \lambda=29 \mathrm{~Hz}$, where $v$ is the velocity of the mobile unit and $\lambda$ is the wavelength. 


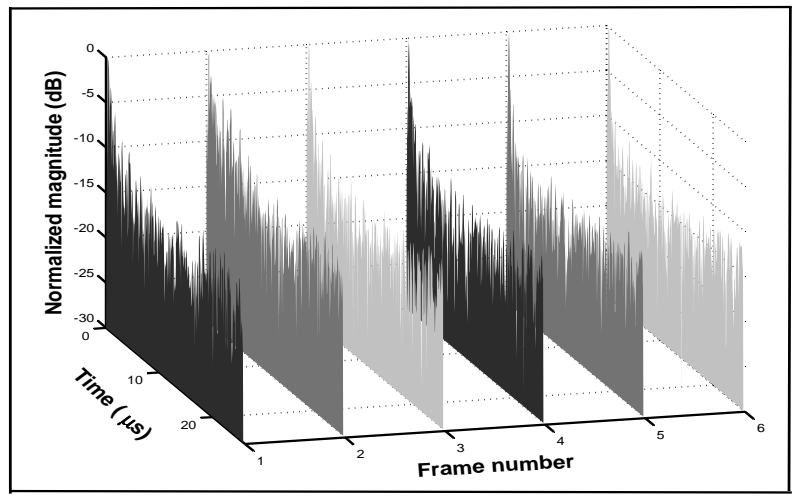

Fig. 3. Channel impulse response over 6 frames: maximum Doppler frequency $\approx 29 \mathrm{~Hz}$ (file: FieldTest-030714-B34; Chemin LineBank)

Power Delay Profile: The Power Delay Profile, PDP, or the delay profile spectrum is determined as the mean power of the channel as function of the path delay $\tau$ [4], [5]. The PDP is generally represented as plot of relative received power as a function of excess delay with respect to a fixed time delay reference. The impulse profile can be characterized by the root mean-square (rms) delay spread $\sigma_{\tau}$, which indicates the time dispersion of a transmitted signal as caused by the multipath. The rms delay spread is defined as:

$$
\sigma_{\tau}=\sqrt{\frac{\sum_{n=1}^{N}\left(\tau_{n}-\bar{\tau}\right)^{2} P\left(\tau_{n}\right)}{\sum_{n=1}^{N} P\left(\tau_{n}\right)}}
$$

where $\bar{\tau}$ is the average delay and it is calculated as:

$$
\bar{\tau}=\frac{\sum_{n=1}^{N} \tau_{n} P\left(\tau_{n}\right)}{\sum_{n=1}^{N} P\left(\tau_{n}\right)}
$$

To process the data files, it is important to eliminate the contributions of the noise at the end (tail) of the PDP. The threshold was chosen to be the sum of the last 200 samples (corresponding to $9 \mu \mathrm{s}$ ) plus 4 times their standard deviation [6]. The signal level below the threshold value was set to zero. Individual relative power delay profiles are shown in figure 4 with the corresponding statistical parameters of the PDP. The measurement is from the same data given in Figure 3. For the first frame, the graphic illustrates a case where 3 components are observed with a relative level 15 $\mathrm{dB}$ below the dominant component. The rms delay spread from frame 2 is higher than from the others, since more multipaths components are considered.

An average power delay profile for a set of $M$ consecutive individual profiles is computed as:

$$
P\left(\tau_{k}\right)=\frac{1}{M} \sum_{i=1}^{M} P_{i}\left(\tau_{k}\right)
$$

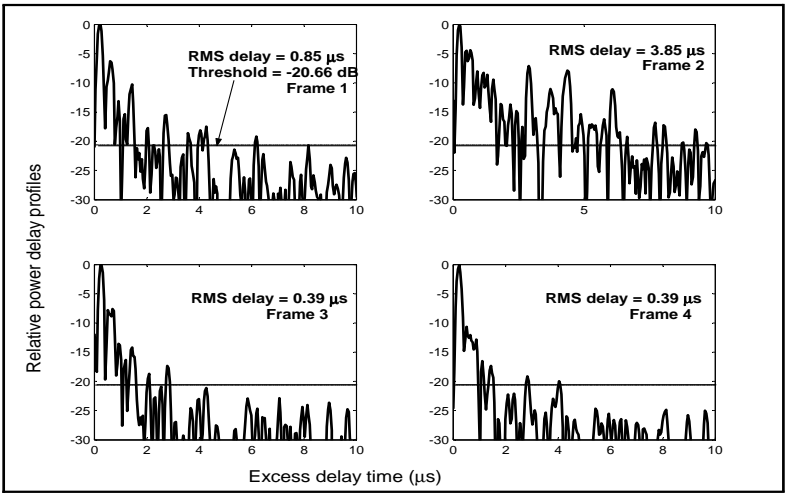

Fig. 4. Power delay profile from 4 consecutive frames: max RMS delay spread $=3.45 \mu$ s (file: FieldTest-030714-B34: Chemin LineBank).

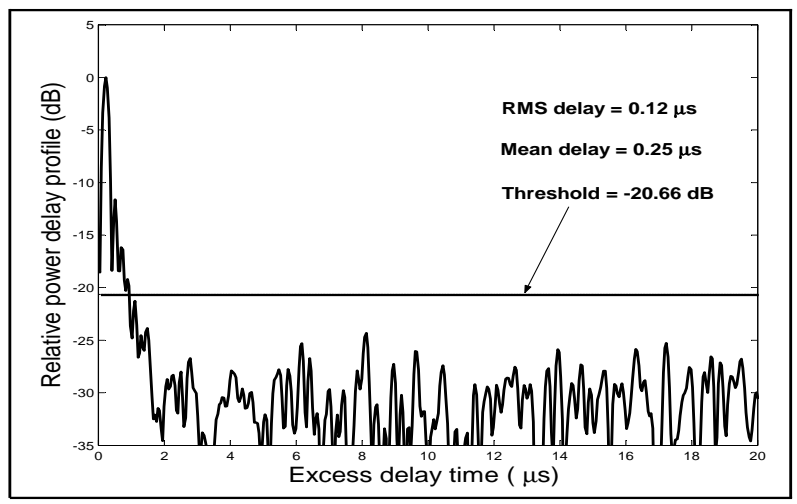

Fig. 5. Average power delay profile over 40 consecutive power delay profiles. (file: FieldTest-030714-B34; Chemin LineBank).

Figure 5 represents an average of 40 consecutive profiles from the Chemin LineBank measurement. It can be seen that the average values for the rms delay spread and the mean delay tends to decrease with the number of the power profiles considered in the calculation.

Table I shows the statistics of the individual and the average rms delay spread over 40 profiles for the two areas considered in this study. Results given in this table concern also the statistics of the PDP derived from the received signals captured from the two antennas. West Avenue and North Drive represent a residential area. Chemin LineBank street and Rideau Road represent an open environment. The individual and average values of the rms delay spread and the mean delay are smaller than one microsecond and the threshold values for differents data are slightly differents.

\section{B. Received signal}

In mobile reception, received signals are affected by short-term and long-term fadings [6] caused by multipath 


\begin{tabular}{||l||c|c|c|c|c||}
\hline Location & $\begin{array}{c}\text { Threshold } \\
(\mathrm{dB})\end{array}$ & \multicolumn{2}{|c||}{$\begin{array}{c}\text { Statistics of } \\
\text { Individual PDP }(\mu \mathrm{s})\end{array}$} & \multicolumn{2}{|c||}{$\begin{array}{c}\text { Statistics of } \\
\text { Average PDPs }(\mu \mathrm{s})\end{array}$} \\
\hline & & Mean delay & RMS delay & Mean delay & RMS delay \\
\hline West Avenue Ch.1 & -21.79 & 0.28 & 0.17 & 0.27 & 0.14 \\
\hline West Avenue Ch.2 & -22.30 & 0.30 & 0.18 & 0.30 & 0.18 \\
\hline \hline North Drive Ch.1 & -20.30 & 0.24 & 0.19 & 0.25 & 0.12 \\
\hline North Drive Ch.2 & -22.14 & 0.24 & 0.12 & 0.29 & 0.18 \\
\hline \hline Rideau Road Ch.1 & -22.71 & 0.24 & 0.97 & 0.24 & 0.09 \\
\hline Rideau Road Ch.2 & -22.78 & 0.26 & 0.10 & 0.25 & 0.10 \\
\hline \hline $\begin{array}{l}\text { Chemin LineBank } \\
\text { Ch.1 }\end{array}$ & -17.19 & 0.78 & 0.80 & 0.34 & 0.24 \\
\hline $\begin{array}{l}\text { Chemin LineBank } \\
\text { Ch.2 }\end{array}$ & -20.66 & 0.54 & 0.85 & 0.25 & 0.12 \\
\hline
\end{tabular}

TABLE I

INDIVIDUAL AND AVERAGE POWER DELAY PROFILE

CHARACTERISTICS.

reflections of a transmitted signal and by the terrain environment. Figure 6 depicts the received signal variations measured at a mobile speed of $30 \mathrm{~km} / \mathrm{h}$. The received envelope signal is from West Avenue street in Ottawa which is considered as a residential area. It was recorded at $9 \mathrm{~h} 11$ $\mathrm{AM}$ and the traffic density was low. This signal is averaged over a sliding window of $10 \mathrm{~ms}$.

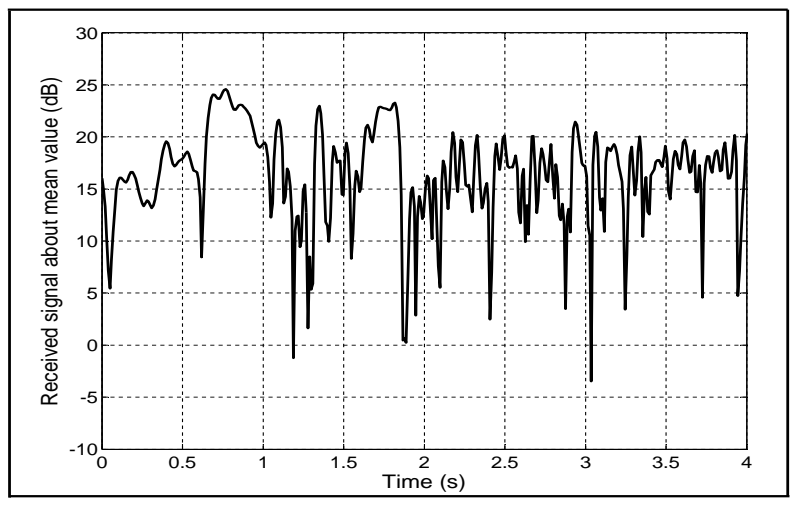

Fig. 6. Received signal strength variations measured at $30 \mathrm{~km} / \mathrm{h}: f_{m}=22$ $\mathrm{Hz}$ (file: FieldTest-030714-A12; West Avenue).

\section{Space diversity results}

The fundamental phenomenon of UHF radio propagation in the urban and suburban mobile radio environment is the existence of multipath with different and varying time delays. Different Doppler shifts are associated with scatter paths arriving at the vehicle from different angles. One way to improve the performance of wireless link is to use space diversity. Diversity exploits the random of radio propagation by finding independent path with a strong signal. The signals received from spatially separated antennas on the mobile may have essentially uncorrelated envelopes for antenna separations of one half wavelength or more [4]. This condition is satisfied in these measurements. In switching diversity [8], the receiver exploits signals that are simultaneously received on each branch. The envelopes of the received signal from the two antennas are compared and the less faded signal is selected instantaneously. Figure 7 shows the envelope of the received signal from each channel and the envelope resulting from the switching between the two branches. This measurement is from the West Avenue and the area is similar as for the last data.

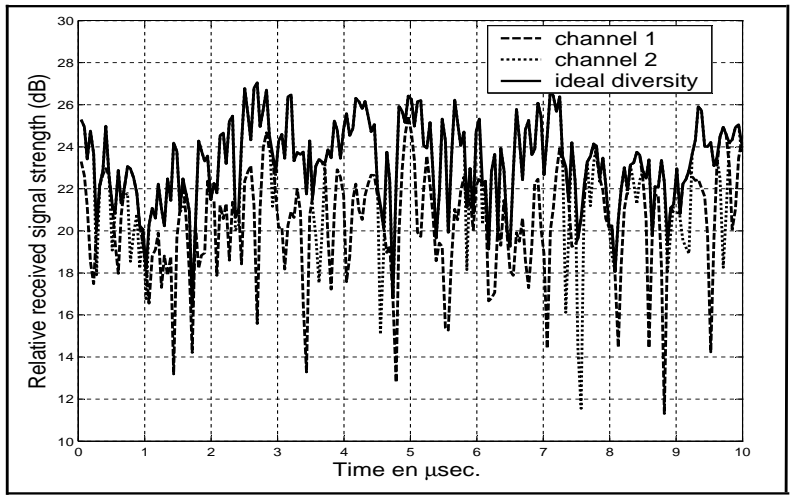

Fig. 7. Switched diversity envelope. (file: FieldTest-030714-A12; West Avenue).

\section{CONCLUSION}

Characteristics of mobile channel has been determined from the ATSC-8VSB received signals collected in the first phase of measurement campain in the region of Ottawa. The statistics of the power delay profile derived from the estimated channel impulse are obtained. The paper report also the effect of the spatial diversity.

\section{REFERENCES}

[1] ATSC, ATSC Digital Television Standard, ATSC standard A/53, september 1995.

[2] P. Peter, R. Burow, G. Faria Lab and Field Tests of mobile applications of $D V B-T$, DVB-T field trials around the world, Geneva, Switzerland, june, 1999.

[3] Y. Wu, X. Wang, K. Salehian, H. Jun, B. Caron Recent performance improvements to the ATSC transmission system, International Broadcasting Convention, pp. 123-133, 1115 September 2003.

[4] T. S. Rappaport, Wireless communications, Prentice-Hill, Inc, 2002.

[5] D. Cox, Delay doppler characteristcs of multipath propagation at 910 $\mathrm{MHz}$ in a suburban mobile radio environment, IEEE Trans. on Ant. and Propagation, VOL. AP-20, No. 5, pp. 625-635, september 1972.

[6] D. C. Kim, H. L. Bertoni, M. Stern Pulse propagation characteristics at $2.4 \mathrm{GHz}$ inside building, IEEE Trans. on Veh. Tech., Vol. 45, NO. 3, pp. 579-592, August 1996.

[7] S. Parsons, The mobile radio propagation channel, Pentech Press, London, 1992.

[8] W. C. Jakes, "A comparison of specific space diversity techniques for reduction of fast fading in UHF mobile radio systems", IEEE Trans. on Veh. Tech., vol. VT-20, No. 4, pp. 81-93, nov. 1971. 